\title{
A Joint Model for Possibly Multivariate Longitudinal End Point in Clinical Cohort Study
}

Yemane Hailu Fissuh*

Department of Statistics, College of Applied Science, Beijing University of Technology, Beijing, China

\begin{abstract}
This paper is motivated in modeling a joint mixed effect model incorporating random effects with independent measurement error for both end points. Both the association in the evolution (AOE) for two or possibly multiple outcomes and evolution in the association (EOA) are expected to be assessed by joint mixed effect model. The proposed model is further trustful of grasping the problem of nonlinearity and absence of normality assumption and in turn is to predict the effect of associated covariates in the progressive evolution of longitudinal outcomes throughout the given time interval. As case study the two outcomes Systolic Blood Pressure (SBP) and Diastolic Blood Pleasure (DBP) of hypertensive patients are considered. The summary statistics of the two end points are included in this context. Thus, the average follow-up is 4.21(0.088) months, the average SBP and DBP of hypertensive patients are $136.12(0.367)$ and $85.13(0.273)$ respectively and the standard deviation of SBP and DBP are 16.21 and 12.06 respectively. Moreover, the average age of the hypertensive patients is $50.63(0.315)$ years old. The values inside the brackets refer the standard errors. Finally, the straight lines on the two plots indicate the normality of the two outcomes. This study suggests for the further work to the extended non-linear mixed effect model for correlated multivariate repeated measure data usually called longitudinal data. Moreover, the study can incorporate the joint model of multivariate longitudinal outcome with time to event outcomes. In order to come up with flexible and robust models, the authors can further extend these models to non-parametric smoothing models of longitudinal endpoints and survival times.
\end{abstract}

Keywords: Blood pressure; End points; Joint mixed effect models; Longitudinal data; Multivariate models

\section{Introduction}

\section{A joint mixed effect model}

In cohort studies the individuals' clinical progress is being checked throughout the entire follow up time. Thus, it is fact to have multiple outcomes in many fields of experimental studies including biomedical, agricultural, public health, epidemiological studies, engineering science and other life science including behavioural sciences researches. Obviously, several questions arise upon the researchers' mind in context that how to model the shared evolution of multivariate longitudinally followed clinical end points. That is the reason for many researchers to be motivated in modelling the correlated outcomes jointly.

In such experimental results the multivariate longitudinal data are unique in the sense that they allow the researchers to study the joint evolution of the outcomes over time. When individual study units are followed from clinical admission until the determination of sure event of interest, loss to follow-up study or the pre-specified time for end of study, whichever condition comes first a progressive study is said to be longitudinal or a follow-up study. Epidemiologists and social scientists usually call the longitudinal studies as cohort studies and panel studies respectively [1]. According to Toh and Hernán [1], once the foremost concern is estimating the causal effect of certain action on the outcome, longitudinal studies are usually ideal and favoured over cross-sectional or non-longitudinal studies for possibly unclear sequential order of treatments and outcome.

- Frequently, multiple response variables are possibly tracked in clinical follow-up of various longitudinal settings. Thus, analysing all settings jointly is much worthier than analysing separately [2]. However, years gone these types of data were being analysed.

- Using simple approaches in which each outcome is analysed separately or by using data reduction approaches like factor analysis and/or principal components to reduce the dimension of several correlated outcomes. Separate analysis approach is practically easier yet it does not able to handle the correlation between longitudinal outcomes and/or other possibly existing features like measurement errors either in one or more responses [3].

- Due to the clear limitations of the two mentioned approaches, since over recent years several researchers have given attention how to model the multivariate outcomes jointly. Consequently, joint mixed effect model for continuous outcomes and joint GEE for discrete longitudinal outcomes have been given high attention to model the joint evolution of two or more longitudinally measured outcomes.

\section{Modelling bivariate continuous longitudinal outcomes jointly}

The commonly used bivariate linear mixed effect models are convenient while analysing longitudinal outcomes of two allied end points. In this paper, the motivation is to model a joint linear mixed effect model incorporating random effects with independent measurement error for both endpoints. Regularly longitudinal data are collected in epidemiological studies specially to study the evolution of biomedical endpoints.

*Corresponding author: Yemane Hailu Fissuh, Department of Statistics College of Applied Science, Beijing University of Technology, Beijing, China, Tel: +8615510370721; E-mail: yemaoroba@gmail.com/yemairob@yahoo.com

Received November 03, 2017; Accepted November 22, 2017; Published November 25, 2017

Citation: Fissuh YH (2017) A Joint Model for Possibly Multivariate Longitudinal End Point in Clinical Cohort Study. J Health Med Informat 8: 292. doi: 10.4172/21577420.1000292

Copyright: ( $) 2017$ Fissuh YH. This is an open-access article distributed under the terms of the Creative Commons Attribution License, which permits unrestricted use, distribution, and reproduction in any medium, provided the original author and source are credited. 


\section{Experimental}

For the elegant analysis the standard statistical packages are available in several software; particularly, freely accessible software $\mathrm{R}$, which is good to model the linear mixed effect model easily handling intra-subject correlation [4]. Furthermore, out of several authors those have modelled the bivariate mixed effect to investigate the joint evolution of two longitudinally measured outcomes, the authors $[5,6]$ recently have published the joint mixed effect models for longitudinal outcomes Systolic Blood Pressure (SBP) and Diastolic Blood Pressure (DBP) of hypertensive patients and Heart Rate (HR) or Pulse Rate (PR) and Respiratory Rate (RR) of congestive heart failure patients respectively.

Thus, the two longitudinally measured endpoints of vector $Y_{i}(t)$, at each occasion which is designed in below are supposed to be modelled jointly.

Suppose the vector $Y_{i}=\left[\begin{array}{l}Y_{1 i} \\ Y_{2 i}\end{array}\right]$ be the response vector for the individual $i$, with $Y_{K i}$ the $n_{k i}$ vector of the end points $k(k=1,2)$ with $\mathrm{n}_{1 \mathrm{i}}=\mathrm{n}_{2 \mathrm{i}}=\mathrm{n}_{\mathrm{i}}$, hence possibly proposed model for bivariate longitudinal data with assumption of Gaussian process is

$$
\left\{\begin{array}{l}
Y_{1 i}(t)=\mu_{1}(t)+a_{1 i}+b_{1 i} t+\varepsilon_{1 i}(t) \\
Y_{2 i}(t)=\mu_{2}(t)+a_{2 i}+b_{2 i} t+\varepsilon_{2 i}(t)
\end{array}\right.
$$

Where, $\mu_{1}(t)$ and $\mu_{2}(t)$ refer to the population means at time $t$. by

The association between the possible evolution of $\mathrm{Y}_{1}$ and $\mathrm{Y}_{2}$ is given

$$
r_{E}=\frac{\operatorname{cov}\left(b_{1}, b_{2}\right)}{\sqrt{\operatorname{var}\left(b_{1}\right) \times \operatorname{var}\left(b_{2}\right)}}=\frac{\sigma_{b_{1} b_{2}}}{\sqrt{\left(\sigma_{b_{1}}^{2} \times \sigma_{b_{2}}^{2}\right)}}
$$

The conceivable marginal association between $1 \mathrm{Y}$ and $2 \mathrm{Y}$ at time $\mathrm{t}$ is given by

$$
\begin{aligned}
& r_{M}(t)=\frac{\operatorname{cov}\left(Y_{1 i}(t), Y_{2 i}(t)\right)}{\sqrt{\operatorname{var}\left(Y_{1 i}(t)\right) \times \operatorname{var} Y_{2 i}(t)}}= \\
& \frac{\sigma_{a_{1} a_{2}}+t \sigma_{a_{1} b_{2}}+\sigma_{a_{2} b_{1}}+t^{2} \sigma_{b_{1} b_{2}}+\sigma_{12}}{\sqrt{\left(\sigma_{a_{1}}^{2}+2 t \sigma_{a_{1} b_{1}}+t^{2} \sigma_{b_{1}}^{2}+\sigma_{1}^{2}\right) \times\left(\sigma_{a_{2}}^{2}+2 t \sigma_{a_{2} b_{2}}+t^{2} \sigma_{b_{2}}^{2}+\sigma_{2}^{2}\right)}}
\end{aligned}
$$

Obviously, it is easy to realize the raise of problems and difficulties in estimating covariance parameters due to the existence of exponential high dimensional covariance parameters with the increase of number of response variables. Therefore, it is true to choose the techniques intuitively.

\section{Case Study}

The case study was done on hypertension patients at Mekelle Ayder Referral Hospital of Mekelle University, Tigray, Ethiopia. The cohort data about SBP and DBP of hypertensive patients is obtained from the hospital. Mekelle University is one of the highly recognized learning and medical training University with enough laboratory equipment which is located in north Ethiopia in Tigray regional state.

\section{Study variables}

- Response variables: Systolic Blood Pressure (SBP) and Diastolic Blood Pressure (DBP)

- Covariates (Independent variables): Age, Sex, Time, Place of Residence, Family history, type of treatment.

\section{Results}

\section{Results of descriptive statistics}

According to the follow up the minimum and maximum age of hypertensive patients are $20 \mathrm{yrs}$ and 86 yrs old respectively. Likewise, the minimum number of SBP and DBP are 90 and 50 respectively and the maximum number of SBP and DBP are 230 and 130 respectively. The maximum follow-up time in months are 18 months and the average follow-up is $4.21(0.088)$ months. Furthermore, the average SBP and DBP of hypertensive patients in this follow-up report are 136.12(0.367) and $85.13(0.273)$ respectively. The total follow-up is 1950 repeated measures.

Additionally, the rounded standard deviation of SBP and DBP are 16.21 and 12.06 respectively. Moreover, the average age of the hypertensive patients is $50.63(0.315)$ yrs old. The values inside the brackets refer the standard errors (Table 1 ).

Both Figures 1 and 2 shows straight line on points indicating that the data fit the normality assumptions.

\section{Discussion}

\section{Summary report}

In these report 354 adolescent hypertensive patients with age greater than or equal to $18 \mathrm{yrs}$, who were on treatment, and who had measured at least three times are considered. The data were extracted from the patient card charts with the guidance of physicians and nurses. The data extraction and clinical follow-up has been taken from August 01, 2017 tod September 30, 2017.

According to the follow up the minimum and maximum age of hypertensive patients are 20 yrs and 86 yrs old respectively. Likewise, the minimum number of SBP and DBP are 90 and 50 respectively and the maximum number of SBP and DBP are 230 and 130 respectively. The maximum follow-up time in months are 18 months and the average follow-up is $4.21(0.088)$ months. Furthermore, the average SBP and

\begin{tabular}{|c|c|c|c|c|c|c|c|}
\hline & \multirow[b]{2}{*}{ N Statistic } & \multirow[b]{2}{*}{ Minimum statistic } & \multicolumn{3}{|c|}{ Descriptive statistics } & \multirow[b]{2}{*}{ Mean std. error } & \multirow[b]{2}{*}{ Std. deviation statistic } \\
\hline & & & Maximum statistic & Sum statistic & Mean statistic & & \\
\hline Time old age & 1950 & 0 & 18 & 8200 & 4.2051 & 0.08783 & 3.87847 \\
\hline Hypertensive patients & 1950 & 20 & 86 & 98719 & 50.6251 & 0.31535 & 13.92568 \\
\hline Systolic blood pressure & 1950 & 90 & 230 & 265430 & 136.1179 & 0.36701 & 16.20661 \\
\hline Diastolic blood pressure & 1950 & 50 & 130 & 166010 & 85.1333 & 0.27303 & 12.05672 \\
\hline Valid N (list wise) & 1950 & & & & & & \\
\hline
\end{tabular}
DBP of hypertensive patients in this follow-up report are 136.12(0.367) and $85.13(0.273)$ respectively. The total follow-up is 1950 repeated measures.

Table 1: Summary statistics. 


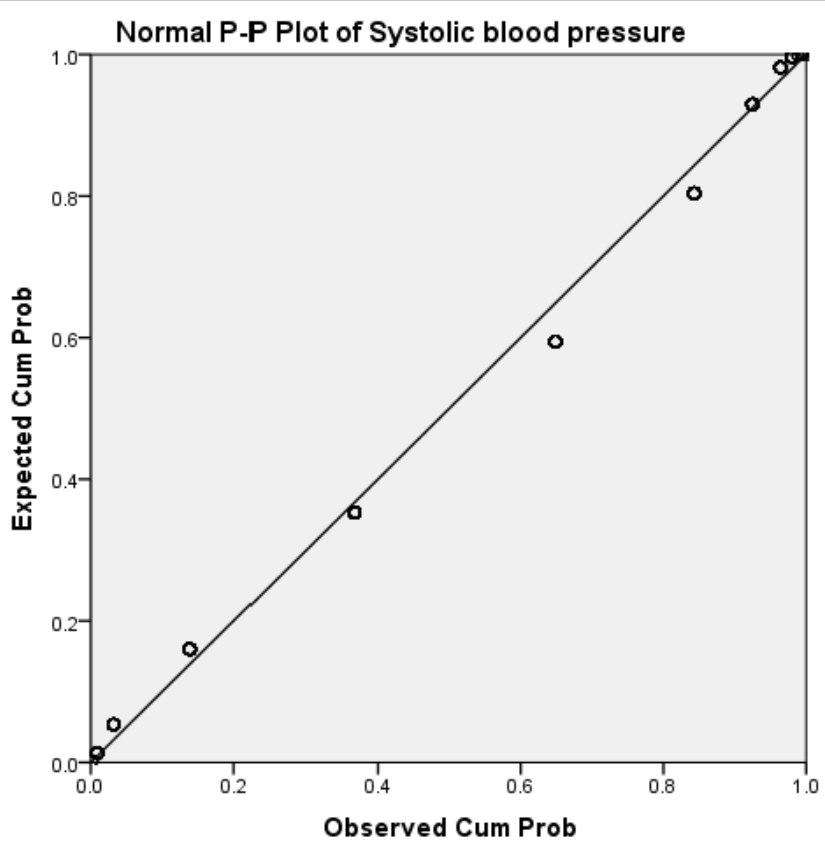

Figure 1: Normal P-P Plot of systolic blood pressure.

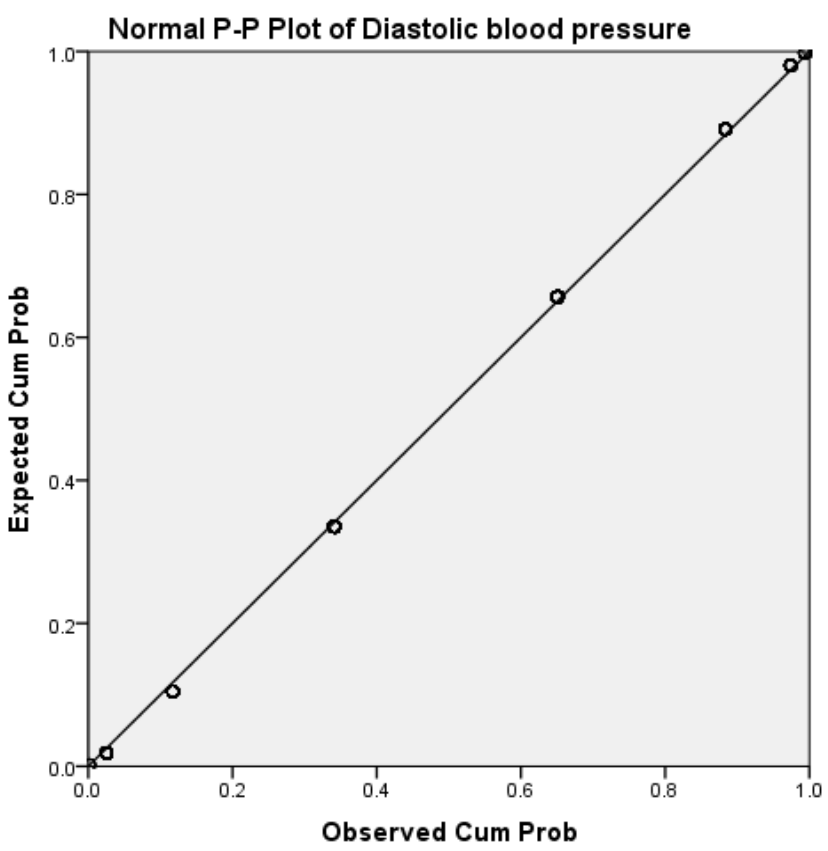

Figure 2: Normal P-P Plot of diastolic blood pressure.

\section{Conclusion}

This study models the mixed effect with the concept of both association in the evolution (AOE) of the two or more responses and the evolution in the associations (EOA) under usual joint linear mixed effects model to grasp the problem of nonlinearity and absence of normality assumptions then to predict the effect of associated factors in the evolution of longitudinal end point through the given time interval.

In all fields of studies such as art, science, engineering and biological or life sciences, especially clinical and epidemiological research, it is usually known to perceive multivariate longitudinal end points. As a result of either experimental or observational outcomes, multivariate longitudinal study initiates the potential researchers to deal with the shared evolution of several outcomes throughout the time. Therefore, the author proposed the joint mixed effect model for multivariate or correlated longitudinal outcomes.

The commonly known assumptions of linear regressions model linearity and normality assumption may not always plausible due to the result of sparse and unbalanced data. Therefore, applying more flexible joint mixed model so-called joint nonlinear mixed effects model for several longitudinal endpoints is more suitable and efficient.

For future work, this can be extended to non-linear mixed effect model for multivariate longitudinal data. Moreover, the study can incorporate the joint model of multivariate longitudinal outcome with time to event outcomes. In order to come up with flexible and robust models, the authors can further extend these models to non-parametric smoothing models of longitudinal end points and survival times.

\section{Acknowledgment}

The author is very grateful to the editors and reviewers for their watchful, insightful and appreciated comments and helpful suggestions that have led to significant improvement of this article.

\section{References}

1. Toh S, Hernán MA (2008) Causal inference from longitudinal studies with baseline randomization. Int J Biostat 4: 1 .

2. Laird NM, Ware JH (1982) Random-effects models for longitudinal data Biomet 38: 963-974.

3. Molenberghs G, Verbeke G (2005) Models for Discrete Longitudinal Data.

4. Littell RC, Milliken GA, Stroup WW, Wolfinger RD (1996) SAS System for Mixed Models.

5. Neupane B, Beyene J (2014) Bivariate linear mixed model analysis to test joint associations of genetic variants on systolic and diastolic blood pressure. BMC 8: S75.

6. Fissuh $\mathrm{YH}$, Muleta $\mathrm{G}$ (2015) A joint model for a longitudinal pulse rate and respiratory rate of congestive heart failure patients: At Ayder Referral Hospital of Mekelle University, Tigray, Ethiopia. J Biom Biostat 6: 260. 